\title{
Correction to: The 2018 UK NHS Digital annual report on the Improving Access to Psychological Therapies programme: a brief commentary
}

Naomi Petra Moller ${ }^{1,2^{*}}$, Gemma Ryan ${ }^{1}$, Jasmine Rollings ${ }^{3}$ and Michael Barkham ${ }^{4}$

\section{Correction to: BMC Psychiatry \\ https://doi.org/10.1186/s12888-019-2235-Z}

Following publication of the original article [1], we have been notified that one of the authors' names is spelled incorrectly. In this Correction the incorrect and correct author name are shown.

Originally the author name has been published as:

- Gemma Ryans

The correct author name is:

- Gemma Ryan

The original article has been corrected.

\section{Author details}

'The British Association for Counselling and Psychotherapy, BACP House, 15, St John's Business Park, Lutterworth LE17 4HB, UK. ${ }^{2}$ School of Psychology, The Open University, Walton Hall, Milton Keynes MK7 6AA, UK. ${ }^{3}$ Department of Psychology, Centre for Comparative and Evolutionary Psychology, University of Portsmouth, University House, Winston Churchill Avenue, Portsmouth PO1 2UP, UK. ${ }^{4}$ Clinical Psychology Unit, Department of

Psychology, The University of Sheffield, Sheffield S10 2TP, UK.

Published online: 17 October 2019

\section{Reference}

1. Moller NP, et al. The 2018 UK NHS Digital annual report on the Improving Access to Psychological Therapies programme: a brief commentary. BMC Psychiatry. 2019;19:252. https://doi.org/10.1186/s12888-019-2235-z.

\footnotetext{
* Correspondence: naomi.moller@bacp.co.uk; naomi.moller@open.ac.uk

${ }^{1}$ The British Association for Counselling and Psychotherapy, BACP House, 15, St John's Business Park, Lutterworth LE17 4HB, UK

${ }^{2}$ School of Psychology, The Open University, Walton Hall, Milton Keynes MK7 6AA, UK

Full list of author information is available at the end of the article
}

(c) The Author(s). 2019 Open Access This article is distributed under the terms of the Creative Commons Attribution 4.0 International License (http://creativecommons.org/licenses/by/4.0/), which permits unrestricted use, distribution, and reproduction in any medium, provided you give appropriate credit to the original author(s) and the source, provide a link to the Creative Commons license, and indicate if changes were made. The Creative Commons Public Domain Dedication waiver (http://creativecommons.org/publicdomain/zero/1.0/) applies to the data made available in this article, unless otherwise stated. 